\title{
Congenital clubfoot treatment with external fixation: Italian experience since 2006
}

\author{
Antonio Memeo, Lucrezia Montanari*, Daniele Priano, Elena Panuccio and Laura Rossi \\ ASST Gaetano Pini-CTO, Centro specialistico Ortopedico Traumatologico Gaetano Pini, P.zza A. Ferrari 1-20122, Milano MI, Italy
}

\begin{abstract}
The term "neglected clubfoot" encompasses several clinical pictures from inveterate clubfoot to relapsed clubfoot or to resistant clubfoot. Treatment with external fixators is a valid option in several case series. In our retrospective study, we wanted to analyze the results obtained in correcting the relapsed /inveterate clubfoot by using external fixators.

In our Unit 101 patients underwent surgical procedures for congenital clubfoot correction from September 2006 to August 2019 . A total of 8 patients (6 male and 2 female) were enrolled in the present study; in two cases the deformity was bilateral so that the total number of feet considered is 10 . The age range of the patients considered at the first treatment of the clubfoot with external fixator ranges from 5 to 10 years with an average age of 7.3 years. The follow-up range is from 13 years to 4 months with an average follow-up of 6.5 years. Of the 10 feet treated with external fixator, 9 were subjected to correction using the Ilizarov apparatus (Ilizarov, TrueLok Orthofix, RRS Dial Medicali) and 1 through Taylor Spatial Frame (TSF Smith \& Nephew).

In evaluating the results of our study, we considered primary and secondary parameters. The primary outcome was therefore good for 7 feet (70\%), discrete for 1 foot and poor in the 2 feet. There is a statistically evidence of all the subdomains of the 2 questionnaires administered (AOFAS and FFI) which demonstrate a clinal and mechanical improvement after treatment. No major complications were recorded among the patients.

The principle of distraction-osteogenesis is a useful option and should be considered as a rescue solution in complex deformities. Its execution by surgeons with considerable experience is crucial. The new hexapod fixators simplify the correction process but remain useful tools in expert hands.
\end{abstract}

\section{Introduction}

Almost all primitive deformities and recurrences of clubfoot can be successfully treated with the Ponseti method, currently considered a gold standard in the treatment of clubfoot [1]. The various surgical strategies are fundamental in relapsed cases.

The term "neglected clubfoot" encompasses several clinical pictures from inveterate clubfoot to relapsed clubfoot or to clubfoot resistant to conservative treatment (often syndromic) [2,3].

The previous treated clubfoot is generally defined as residual or recurrent; the residual deformities are those that have never been corrected, while the recurrent deformities are those that had been completely corrected previously and that reappear at a distance.

The late recurrence of clubfoot deformities, it is important to perform a neurological evaluation to rule out previously undiagnosed neurological pathologies.

Another entity is the inveterate clubfoot; by definition, these feet have never been treated and often show severe callus deformity on the lateral surface of the foot or at the level of the head of the talus. These feet do not have extensive scarring and/or postoperative retractions, and many can be treated with the Ponseti method, even in older children [4].

Treatment with external fixators is a valid option in several case series [5-7].

The main advantage of this technique is the high success rate compared to common complications in the classic surgical treatment of these deformities. The most common complications are related to soft tissues, vascularization, surgical wounds, the tendency to recurrence, as well as those deriving from the use of open techniques (arthrodesis and bone resections) in skeletally immature patients. There is no consensus on when to consider an inveterate or relapsed clubfoot as not suitable for conservative treatments $[5,8]$.

The criteria for external fixation treatment include: adult patients or children over 5 years of age; patients with non-reducible feet after manipulations and serum chalks; patients who have undergone three unsuccessful surgical treatments; syndromic cases (arthrogryposis, sequelae of neuromuscular diseases, Marfan syndrome); patients undergoing complicated open surgical treatments with soft tissue necrosis and recurrence $[7,9,10]$.

A different grouping method is the classification proposed by Dimeglio et al. [11] In this classification, patients with grades III and IV would be eligible for treatment [12].

The aim of the treatment is to obtain a plantigrade and painless foot, with dimensions like the contralateral foot.

The classic approach to treat complex foot deformities involves one-step surgical correction with multiple osteotomies or wedge

*Correspondence to: Lucrezia Montanari, ASST Gaetano Pini-CTO, Centro specialistico Ortopedico Traumatologico Gaetano Pini, P.zza A. Ferrari 1-20122, Milano MI, Italy, E-mail: 1.montanari89@gmail.com

Key words: Clubfoot, Hexapod external fixation, paediatric foot

Received: April 01, 2020; Accepted: April 07, 2020; Published: April 20, 2020 
resections, soft tissue release and arthrodesis. The main disadvantage of this approach is the consequent shortening of the foot due to the large bone resection necessary for the correction. In addition, it is an invasive surgery, subject to several complications including wound healing problems, neurovascular lesions and incomplete correction of deformity.

The major advantage of the distraction-osteogenesis principle is that it allows correction with a foot stretch rather than a shortening. In addition, the correction forces can be dynamically adjusted during treatment based on the response of the deformity to the correction. The disadvantages are like those of any other prolonged treatment with an external fixator, including prolonged discomfort for the patient, infections via the pins and muscle or joint contractures $[8,13,14]$.

In our retrospective study, we wanted to analyze the results obtained in correcting the relapsed /inveterate clubfoot by using an external fixator in our cases, collected from September 2006 to August 2019.

\section{Materials and methods}

In the Orthopedics and Traumatology Pediatric Unit of Gaetano Pini Hospital in Milan, 101 patients underwent surgical procedures for congenital clubfoot from September 2006 to August 2019. Of these, 6 patients had a recurrence of deformity which was surgically treated by an external fixator. Among these 6 patients, 3 had a second recurrence of the deformity at 5,2 and 1.5 years respectively after the removal of the external fixator. 2 of these relapses were treated with an external fixator. 1, presenting only a relapse of the adduction component of the forefoot, was subjected to a medial capsulotomy associated with a wedge subtraction wedge osteotomy.

In our study we also included 2 cases of severe inveterate clubfoot (grade III according to Dimeglio's classification)[11]: one patient (female) came to our attention at 8 years, presenting a bilateral congenital clubfoot while the other patient (male) is came to our attention at the age of 6 and suffering from unilateral congenital clubfoot (left). Both had never been treated previously.

All 8 patients had a varus equine clubfoot with a supine adduct.

At our operating unit, clubfoot treatment involves the following standardized steps:

\section{- Parent diagnosis and counseling}

- initial bloodless treatment with progressive chalks as per Ponseti method

- possible Achilles elongation at 3 months or when the child's $6 \mathrm{~kg}$ body weight is reached

- any accessory times of posterior / medial capsulotomy or tenotomy of the long flexor of the big toe / plantar fascia

- tutoring up to $4-5$ years

- monitoring of relapses

In case of recurrence, children under 5 years of age are eventually treated with accessory surgical times and Achilles lengthening in association with corrective chalks.

In relapses over 5 years of age or in the case of inveterate severe clubfoot over 5 years of age, in our unit, we use the surgical correction technique using an external fixator.

\section{The inclusion criteria used in this study are:}

- clubfoot unresponsive to treatment with serial manipulations and chalks clubfoot relapsed after surgical treatment

- clubfoot classified as Grade III / IV according to Dimeglio's classification

- age $>5$ years

- syndromic patients

According to these inclusion criteria, 8 patients were selected, 6 males (75\%) and 2 females (25\%).

The age range of patients considered at the first treatment of clubfoot with external fixator ranges from 5 to 10 years with an average age of 7.3 years. The two cases retracted with external fixator for recurrence, after the first treatment, presented respectively 7 and 13 years.

Of the 8 patients considered in study 2 are of the group of club feet associated with syndromes: 1 arthrogryposis and 1 Soto's syndrome.

Of the 8 patients considered in the study, 4 had bilateral congenital clubfoot but of these only 2 had recurrence with the need for correction by external fixator bilaterally; the total therefore of the feet subjected to treatment with external fixator is 10 to which the 2 feet relapsed after the first treatment with fixator and treated again with external fixator must be added.

In all 6 patients treated with monolateral external fixator, the left side was the affected side. The follow-up range is from 13 years to 4 months with an average follow-up of 6.5 years.

All patients treated for recurrence of the deformity had previously undergone surgical procedures (see table); in all cases interventions had already been performed on soft tissues and in 2 cases procedures had also been performed on the skeleton: 1 cuboid subtraction wedge osteotomy and 1 cuboid subtraction wedge osteotomy in combination with addition wedge osteotomy of the 1st wedge-shaped.

The time interval from the last surgical procedure to the placement of the external fixator ranges from 13 months (1 year and 1 month) to 107 months (8 years and 11 months) with an average interval of 47 months ( 3 years and 11 months).

Of the 10 feet treated with external fixator, 9 were subjected to correction using the Ilizarov apparatus (Ilizarov, TrueLok Orthofix, RRS Dial Medicali) and 1 through Taylor Spatial Frame (TSF Smith \& Nephew) which represents the first of the hexapod external fixator models. All 3 feet relapsed after the first external fixator treatment had been treated with Ilizarov's apparatus ( 2 TrueLok and 1 RRS); of the 2 retracted with external fixation, 1 was retracted with the Ilizarov apparatus (RRS) while the other was subsequently treated with TSF by combining the correction of the residual deformity at the foot with that of the curved tibia.

A thorough clinical examination was performed in all patients to rule out any other associated lower limb deformity. Furthermore, walking was analyzed to exclude the presence of any muscle imbalances. Standard anterior-posterior and lateral radiographs of the feet were used to confirm the diagnosis and evaluate the skeletal anatomy.

The assembly of the Ilizarov external fixator requires (Figure 1).

- two rings at the level of the leg made integral with the skeletal plane by means of threads/chips e connected by bars 
- a half ring at the hindfoot stabilized with wires and positioned to mimic the equine and varus deformity of the calcaneus and possibly adduction

- a half ring at the forefoot stabilized with metatarsal threads

- assembly of the threaded correction bars

- finger infibulation with Kirschner wires and assembly of the wire connection bar with the front half-ring

The assembly of the external hexapod fixator requires (Figure 2).

- a ring proximal to the leg

- a tibial transepiphyseal wire connected to the proximal ring

- a ring distal to the foot

- an olive thread in the talus which in the first phase of correction of the internal torsion and varus of the hindfoot is connected to the proximal ring; in the second phase of correction of equinism, this wire is repositioned and connected to the distal ring

- six bars of adjustable length

The procedures were performed under general anesthesia.

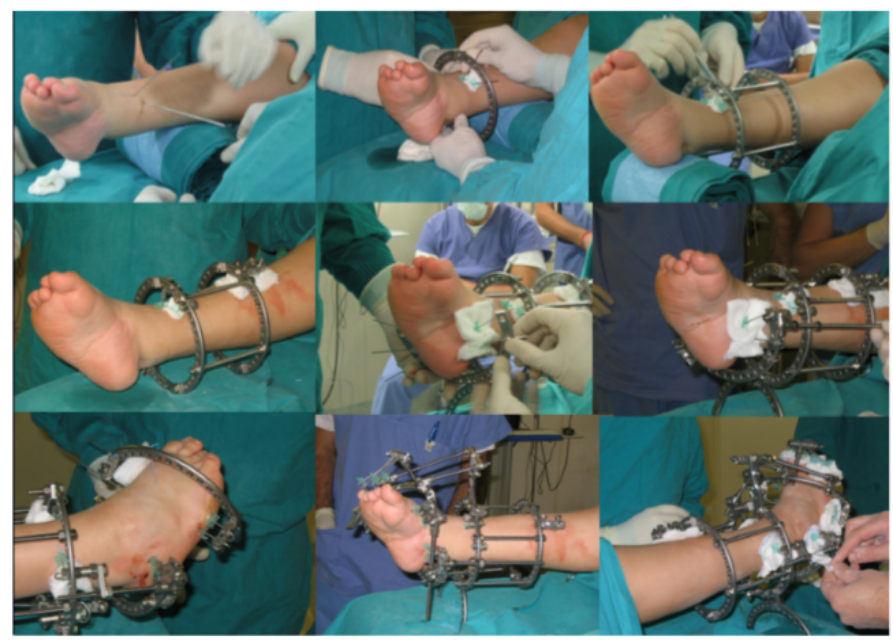

Figure 1. Assembly of the Ilizarov external fixator

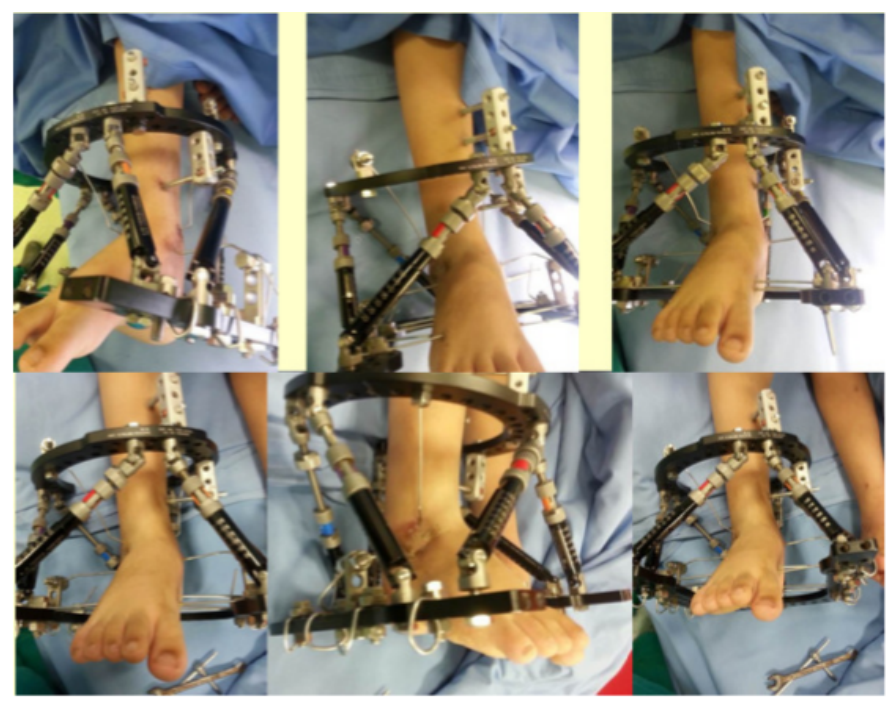

Figure 2. Assembly of the Hexapod external fixator
In 4 out of 10 feet accessory procedures were performed on soft tissues: Achilles elongation and plantar fasciotomy in 2 relapsed club feet and in 1 relapsed club foot the posterior capsulotomy was also associated; Achilles elongation, tenotomy of the long flexor from the big toe, posterior tibio-astragalic and subtalagal capsulotomy and release of the plantar fascia in the inveterate unilateral case.

The standard daily care of the wires and chips is started on the 1st postoperative day, while the lengthening/correction program is started on the 3rd-5th postoperative day. Upon discharge from the hospital, patients and their caregivers were trained on how to perform daily stretch/correction and home care.

The time interval with the external fixator in place ranges from 1 to 6 months with an average of 3.6 months, depending on the severity of the deformity to be corrected. In general, once the correction is obtained, the fixator is kept in place for about 4 weeks before removal.

The external fixator is then removed in sedation and a pinstripe boot is packaged to be kept for 4-6 weeks and then replaced by removable braces during the day to perform physical therapy.

\section{Results}

In evaluating the results of our study, we considered primary and secondary outcome parameters.

Among the primary parameters we took into consideration:

- plantigrade support

- pain absent or limited (VAS score $<2$ after activity)

- absence of recurrence at follow-up

- patient satisfaction (yes/no).

The result was therefore defined as good, discreet and poor based on these criteria: when all 4 criteria were met, the result was classified as good; when 3 out of 4 criteria were met, the result was classified as discrete; when 2 out of 4 criteria were achieved, the result was classified as poor.

As secondary outcome parameters of the study we considered the American Orthopedic Foot and Ankle Score (AOFAS) and the Foot Function Index (FFI) administered with the help of Parents in the preand post-operative period.

Originally published in 1994 on Foot and Ankle International, the American Orthopedic Foot and Ankle Society score (AOFAS) provides a standardized assessment of the clinical status of the ankle and hindfoot. Incorporates both subjective and objective information: patients report their pain and doctors evaluate alignment; both then work together to complete the functional part. Scores range from 0 to 100 points [15].

The Foot Fuction Index was published in 1991 in the journal Physical Therapy; its original version included 23 questions, which covered three sub-scales of foot function: pain, disability and limitation of activity [16]. The score is based on a visual analogue scale with 10 intervals. Scores are calculated for each of the sub-scales, as well as a total score (average of all secondary scales). The version of FFI that we used is the one subsequently reduced to 17 questions, according to the observations of Venditto et al., 2015, which reported a very high percentage of patients ( $>90 \%$ ) with null or non-applicable answers to the 6 questions removed [17]. 
Data analysis was performed with the Jamovi statistical package version 1.1.7. (Https://www.jamovi.org/)

For the descriptive analysis of continuous variables, the main position, dispersion and shape indices were calculated, including, mean, median, fashion, variance, standard deviation, interquartile difference, minimum, maximum, range, asymmetry coefficient, kurtosis coefficient. Where relevant, standard errors and related $95 \%$ confidence intervals were also reported. Parametric tests (Student's $\mathrm{t}$ test) and nonparametric tests (Mann-Whitney $\mathrm{U}$ test or Wilcoxon test) were used for comparisons between groups relating to continuous variables.

The results were considered statistically significant for a p-value of less than $5 \%(\mathrm{p}<0.05)$.

A total of 8 patients ( 6 male and 2 female) were enrolled in the present study; in two cases the deformity was bilateral so that the total number of feet considered was 10 . The two relapsed cases were assessed pre and post both as regards the first treatment with fixator and as regards the further treatment of recurrence with fixator; for the purposes of statistical analysis, being otherwise too small as a sample, only the values of start of treatment and of were considered end of the overall treatment (then also the second treatment with the fixator).

The average age of patients undergoing treatment with an external fixator was 7.3 years (range 5-10 years) and the average follow-up is 6.5 years (range 13 years - 4 months).

All patients had complex rigid foot deformity, 2 in association with syndromes (arthrogryposis and S. di Sotos), 4 as sequelae of an idiopathic clubfoot already undergoing previous treatments and 2 as an inveterate congenital idiopathic clubfoot.

Except for the two patients with inveterate congenital clubfoot, all the others had previously undergone capsular release surgery, tendon lengthening or osteotomies with unsatisfactory results.

The average time interval between the last surgery and the start of the treatment with fixator is 47 months (3 years and 11 months; range 13-107 months).

Soft tissue surgical procedures were performed in 4 out of 10 , in association with the placement of the external fixator. The average hospitalization time was 5.7 days (range 3-16 days).

\section{Primary outcome parameters}

Plantigrade support, at the end of the treatment with the fixator, was achieved in all patients, however 2 out of 10 feet (1 and 3) (20\%) presented a recurrence of equinism after 5 and 2 years respectively, and the plantigrade support criterion in these two cases was therefore considered as not satisfied for the purpose of the overall calculation of the primary outcome.

In 9 out of 10 feet (90\%) there was a marked improvement in algic symptoms with a VAS <2 after activity; only in 1 foot (1) was a VAS $>2$ recorded with the activity.

A recurrence occurred in 3 out of 10 feet considered in our study: in 2 feet there was a recurrence of equinism in association with a tibial deformity ( 1 recurved and 1 intra-rotation) and underwent further treatment with external fixator; in 1 foot there was a recurrence of the adduction which was corrected with osteotomy.

The primary outcome was therefore good for 7 out of 10 feet $(70 \%)$, discrete for 1 foot (due to the recurrence of the adduction) and poor in the 2 feet which were then subjected to a new treatment with external fixator. The primary outcomes following retreatment with external fixator were good in both patients (Table 1).

\section{Secondary outcome parameters}

The results of the 2 questionnaires administered, AOFAS and FFI, were collected. For the statistical analysis, given the narrowness of the "recurrence" sample (only 2 cases retracted with fixator), the overall values of their treatment were considered, ie the values of AOFAS and FFI initial pre-treatment and the values of AOFAS and FFI post second definitive treatment with external fixator. Statistical analysis showed a statistically significant improvement of all subdomains of both questionnaires (Tables 2 and 3 ).

\section{Complications}

No major complications were recorded among the patients included in the following study. minor complications include those typically associated with external fixation treatments, such as superficial transfection infections, contractures and joint stiffeners that have been treated with adequate antibiotic coverage and physical therapy respectively when removing the external fixation device.

\section{Discussion}

The treatment initially proposed in the case of club feet is the conservative method according to Ponseti and should begin as soon as possible. Conservative treatment can be accompanied by minimally invasive surgical procedures such as percutaneous tenotomy of the Achilles tendon to correct a resistant equinism. If a good correction is not obtained within 6 months, the probability of having to intervene with the release of the soft tissues increases. The recurrence or persistence of deformity is reported in many articles [18-20].

In the Orthopedics and Traumatology Pediatric Unit of Gaetano Pini Hospital in Milan, 101 patients underwent surgical procedures for congenital clubfoot from September 2006 to August 2019.

Of these, 6 patients (6\%) experienced a recurrence of deformity which was surgically treated by an external fixator.

Of the total 10 feet considered in this study, 3 had a recurrence after treatment with an external fixator (30\%).

Recurrence can be caused by multiple factors specific to the disease, by an incorrect diagnosis, by inadequate treatment or by poor postoperative management $[6,8,9,21]$.

Of the 3 relapsed feet, the 2 that were retracted with an external fixator, had already undergone 2 surgical procedures on the soft tissues and the recurrence of the foot deformity was associated with a tibial

Table 1. Primary outcome parameters

\begin{tabular}{|c|c|c|c|c|c|}
\hline S.no & $\begin{array}{c}\text { Plantigrade } \\
\text { support }\end{array}$ & Pain & Recurrence & Satisfaction & Outcome \\
\hline $\mathbf{1}$ & No & Yes & Yes & No & Poor \\
\hline $\mathbf{2}$ & Yes & No & No & Yes & Good \\
\hline $\mathbf{3}$ & No & No & Yes & No & Discreet \\
\hline $\mathbf{4}$ & Yes & No & No & Yes & Good \\
\hline $\mathbf{5}$ & Yes & No & No & Yes & Good \\
\hline $\mathbf{6}$ & Yes & No & No & Yes & Good \\
\hline $\mathbf{7}$ & Yes & No & Yes & Yes & Discreet \\
\hline $\mathbf{8}$ & Yes & No & No & Yes & Good \\
\hline $\mathbf{9}$ & Yes & No & No & Yes & Good \\
\hline $\mathbf{1 0}$ & Yes & No & No & Yes & Good \\
\hline
\end{tabular}


Table 2. Statistical Analysis FFI test

\begin{tabular}{|c|c|c|c|c|c|c|}
\hline \multicolumn{7}{|c|}{ Paired Samples T-Test } \\
\hline & & & Statistic & df & $\mathbf{p}$ & Cohen's d \\
\hline \multirow{2}{*}{ D_FFI PRE } & \multirow{2}{*}{ D_FFI POST } & Student's t & 6.99 & 9.00 & $<.001$ & 2.21 \\
\hline & & Wilcoxon W & 55.0 & & 0.006 & 2.21 \\
\hline \multirow{2}{*}{ DIS_FFI PRE } & \multirow{2}{*}{ DIS_FFI POST } & Student's t & 8.98 & 9.00 & $<.001$ & 2.84 \\
\hline & & Wilcoxon W & 55.0 & & 0.002 & 2.84 \\
\hline \multirow{2}{*}{ L_FFI PRE } & \multirow{2}{*}{ L_FFI POST } & Student's t & 6.20 & 9.00 & $<.001$ & 1.96 \\
\hline & & Wilcoxon W & 55.0 & & 0.006 & 1.96 \\
\hline \multirow{2}{*}{ S_T_FFI PRE } & \multirow{2}{*}{ S_T_FFI POST } & Student's t & 8.67 & 9.00 & $<.001$ & 2.74 \\
\hline & & Wilcoxon W & 55.0 & & 0.002 & 2.74 \\
\hline \multicolumn{7}{|c|}{ Descriptives } \\
\hline & $\mathbf{N}$ & Mean & Median & SD & SE & \\
\hline D_FFI PRE & 10 & 20.10 & 14.50 & 8.85 & 2.799 & \\
\hline D_FFI POST & 10 & 3.10 & 3.00 & 2.77 & 0.875 & \\
\hline DIS_FFI PRE & 10 & 54.70 & 56.50 & 18.60 & 5.880 & \\
\hline DIS_FFI POST & 10 & 7.30 & 6.00 & 6.25 & 1.978 & \\
\hline L_FFI PRE & 10 & 16.40 & 16.50 & 7.96 & 2.517 & \\
\hline L_FFI POST & 10 & 2.10 & 2.50 & 1.60 & 0.504 & \\
\hline S_T_FFI PRE & 10 & 91.20 & 86.50 & 33.65 & 10.642 & \\
\hline S_T_FFI POST & 10 & 3.10 & 3.00 & 2.77 & 0.875 & \\
\hline
\end{tabular}

Table 3. Statistical Analysis FFI test.

\begin{tabular}{|c|c|c|c|c|c|c|}
\hline \multicolumn{7}{|c|}{ Paired Samples T-Test } \\
\hline & & & Statistic & df & $\mathbf{p}$ & Cohen's d \\
\hline \multirow{2}{*}{ D_AOFAS_P } & \multirow{2}{*}{ D_AOFAS POST } & Student's t & -4.02 & 9.00 & 0.003 & -1.27 \\
\hline & & Wilcoxon W & $0.00^{\mathrm{a}}$ & & 0.007 & -1.27 \\
\hline \multirow{2}{*}{ F_AOFAS_P } & \multirow{2}{*}{ F_AOFAS POST } & Student's t & -15.53 & 9.00 & $<.001$ & -4.91 \\
\hline & & Wilcoxon W & 0.00 & & 0.006 & -4.91 \\
\hline \multirow{2}{*}{ A_AOFAS_P } & \multirow{2}{*}{ A_AOFAS POST } & Student's t & $\mathrm{NaN}^{\mathrm{b}}$ & & & \\
\hline & & Wilcoxon W & 0.00 & & 0.002 & $-\operatorname{Inf}$ \\
\hline \multirow{2}{*}{ ST_AOFAS_P } & \multirow{2}{*}{ ST_AOFAS POST } & Student's t & -11.37 & 9.00 & $<.001$ & -3.60 \\
\hline & & Wilcoxon W & 0.00 & & 0.002 & -3.60 \\
\hline \multicolumn{7}{|c|}{ Descriptives } \\
\hline & $\mathbf{N}$ & Mean & Median & SD & SE & \\
\hline D_AOFAS_P & 10 & 23.00 & 30.00 & 13.37 & 4.23 & \\
\hline D_AOFAS POST & 10 & 38.00 & 40.00 & 4.22 & 1.33 & \\
\hline F_AOFAS_P & 10 & 4.20 & 0.00 & 8.56 & 2.71 & \\
\hline F_AOFAS POST & 10 & 47.70 & 49.50 & 3.27 & 1.03 & \\
\hline A_AOFAS_P & 10 & 0.00 & 0.00 & 0.00 & 0.00 & \\
\hline A_AOFAS POST & 10 & 5.00 & 5.00 & 0.00 & 0.00 & \\
\hline ST_AOFAS_P & 10 & 27.20 & 30.00 & 18.79 & 5.94 & \\
\hline ST_AOFAS POST & 10 & 90.70 & 94.50 & 6.70 & 2.12 & \\
\hline
\end{tabular}

a 1 Pair(s) of values were tied. ${ }^{\text {b }}$ data were essentially constant

deformity (recurved and intra-rotation of the tibia, respectively). 1 of these 2 patients had foot deformity in association with a picture of arthrogryposis. Therefore, when analyzing the recurrence rate of $30 \%$ of our study, we must consider the severity and complexity of the cases that are reserved for treatment with an external fixator.

Conventional treatment of relapsed clubfoot consists in performing repeated or more radical releases of soft tissues, tendon transference or osteotomies $[2,14]$. These procedures can cause stiffness of the foot or reduction of the size of a foot which is already smaller than the contralateral unaffected in case of monolaterality of the pathology.

Tissues, subjected to gradual traction at a speed of about $1 \mathrm{~mm}$ per day, becomes active and regenerative, thus allowing to remodel bones and soft tissues. The application of this principle in children, in association or not with surgical procedures on soft or skeletal tissues, leads to the correction of many complex deformities of the foot.
In literature, the combination of soft tissue release and gradual correction with the Ilizarov method is presented as a safe procedure in cases where conservative treatment alone cannot be successful $[9,12]$.

The treatment with external fixator allows a simultaneous and three-dimensional correction of the deformity of the foot without causing a shortening of it as often happens with conventional surgical treatments.

The lengthening and progressive correction also favor a gradual elongation of the vessels, nerves, muscles, connective tissue and skin, thus reducing the risk of neurovascular damage, skin necrosis and secondary infections.

The treatment of relapses of congenital clubfoot or inveterate congenital clubfoot over 5 years with circular external apparatus has several advantages: it is a percutaneous technique and the correction occurs gradually on several anatomical planes. The correction can also 
be monitored and therefore corrections are constantly allowed without however compromising the possibility of any further subsequent surgical treatments should the need arise.

The disadvantages related to this method are typical of the treatment with an external fixator: prolonged discomfort for the patient, for which careful and particular collaboration by parents is essential; superficial infections by means of threads and chips, for which an adequate training of the caregivers in the care of the fixator is important; muscle contractures or residual joint stiffness, so subsequent physical therapy is essential $[5,12,22]$.

As primary parameters for the evaluation of the treatment with external fixator we have taken into consideration: the achievement of a plantigrade support, the absence of pain with the activity (VAS score $<2$ after activity), the absence of recurrence at follow-up and the satisfaction of the patient and parents at the end of the treatment. Of the 10 feet considered in the present study: 7 presented a good primary outcome (70\%), 1 presented a discrete primary outcome (due to the recurrence of adduction), 2 presented a poor primary outcome (20\%) for recurrence 5 and 2 years after the deformity, they were then subjected to a new treatment with an external fixator with a marked improvement in the primary outcome (good for both patients after the second treatment with an external fixator).

As secondary parameters for the evaluation of the treatment we administered two questionnaires, AOFAS and FFI with reference respectively to the period preceding the treatment with external fixator (PRE) and following the treatment (POST).

The statistical analysis of the pain, function and alignment subdomains and the total score of the AOFAS questionnaire revealed a statistically significant difference between the PRE and POST treatment values.

A statistical analysis of the pain, disability, limitation and total score of the FFI questionnaire revealed a statistically significant difference between the PRE and POST treatment values for all.

No major complications were recorded among the patients included in the following study: among the minor complications we found superficial transfection infections, contractures and joint stiffeners which were respectively treated with adequate antibiotic coverage and physical therapy when the external fixation device was removed.

We recognize as a limit of this study the relatively small number of patients included, however it must be related to the number of patients with complex foot deformities who respect the inclusion criteria of the study and require this type of surgical treatment; we find it important to underline, given the nature of the disease to recur, the important average of our 6.5-year follow-up (range 13 years - 4 months).

\section{Conclusion}

The statistical analysis of the pain, function and alignment subdomains and the total score of the AOFAS questionnaire revealed a statistically significant difference between the PRE and POST treatment values.

A statistical analysis of the pain, disability, limitation and total score of the FFI questionnaire revealed a statistically significant difference between the PRE and POST treatment values for all.

No major complications were recorded among the patients included in the following study: among the minor complications we found superficial transfection infections, contractures and joint stiffeners which were respectively treated with adequate antibiotic coverage and physical therapy when the external fixation device was removed.

We recognize as a limit of this study the relatively small number of patients included, however it must be related to the number of patients with complex foot deformities who respect the inclusion criteria of the study and require this type of surgical treatment; we find it important to underline, given the nature of the disease to recur, the important average of our 6.5-year follow-up (range 13 years - 4 months).

The main advantage of this technique is the high success rate compared to common complications in the classic surgical treatment of these deformities; other advantages related to this method: it is a "closed sky" technique, the classic surgical approach is not necessary and the correction takes place gradually on several anatomical planes, the correction can also be monitored and therefore "additional" corrections are constantly allowed without however compromise the possibility of any further subsequent surgical treatments if necessary.

However, our study also shows that this treatment is plagued by a $20 \%-30 \%$ failure rate, with some patients needing other procedures at the end of the treatment. Patients and their parents must be carefully informed about all the options available before the intervention and must be informed of the possibility of failure and the prolonged treatment times that this type of intervention requires. The distractionosteogenesis principle is a useful option and should be considered as a rescue solution in complex deformities, it turns out however, its execution by surgeons with considerable experience in its application is crucial. The new hexapod fixators, of which we used the precursor (TSF) in our study, simplify the correction process, but remain useful tools in expert hands.

\section{Conflict of interest}

The author(s) declare that they have no competing interests

\section{References}

1. Sanzarello I, Nanni M, Faldini C (2017) The clubfoot over the centuries. J Pediatr Orthop B 26: 143-151. [Crossref]

2. Eidelman M, Kotlarsky P, Herzenberg JE (2019) Treatment of relapsed, residual and neglected clubfoot: Adjunctive surgery. J Child Orthop 13: 293-303. [Crossref]

3. Smith WG (2015) Interventions for congenital talipes equinovarus (clubfoot). Paediatr Child Health 20: 307-308. [Crossref]

4. Balasankar G, Luximon A, Al-Jumaily A (2016) Current conservative management and classification of club foot: A review. J Pediatr Rehabil Med 9: 257-264. [Crossref]

5. Riganti S, Coppa V, Nasto LA, Di Stadio M, Calevo MG, et al. (2019) Treatment of complex foot deformities with hexapod external fixator in growing children and young adult patients. Foot Ankle Surg 25: 623-629. [Crossref]

6. Malizos KN, Gougoulias NE, Dailiana ZH, Rigopoulos N, Moraitis T (2008) Relapsed clubfoot correction with soft-tissue release and selective application of Ilizarov technique. Strategies Trauma Limb Reconstr 3: 109-117. [Crossref]

7. Ganger R, Radler C, Handlbauer A, Grill F (2012) External fixation in clubfoot treatment - A review of the literature. J Pediatr Orthop 21: 52-58. [Crossref]

8. Ettl V, Kirschner S, Krauspe R, Raab P (2009) Midterm results following revision surgery in clubfeet. Int Orthop 33: 515-520. [Crossref]

9. Bradish CF, Noor S (2000) The Ilizarov method in the management of relapsed club feet. J Bone Joint Surg Br 82: 387-391. [Crossref]

10. Wang XJ, Chang F, Su YX, Wei XC, Wei L (2018) Ilizarov technique combined with limited adjunctive surgical procedures for correction of relapsed talipes equinovarus in children. J Int Med Res 46: 802-810. [Crossref] 
11. Diméglio A, Bensahel H, Souchet P, Mazeau P, Bonnet F (1995) Classification of clubfoot. J Pediatr Orthop B 4: 129-136. [Crossref]

12. Fernandes RMP, Mendes MD dos S, Amorim R, Preti MA, Sternick MB, et al. (2016) Surgical treatment of neglected clubfoot using external fixator. Rev Bras Ortop 51: 501-508. [Crossref]

13. van Bosse HJ (2013) Treatment of the neglected and relapsed clubfoot. Clin Podiatr Med Surg 30: 513-530. [Crossref]

14. Pohl M, Nicol RO (2003) Transcuneiform and opening wedge medial cuneiform osteotomy with closing wedge cuboid osteotomy in relapsed clubfoot. J Pediatr Orthop 23: 70-73. [Crossref]

15. Kitaoka HB, Alexander IJ, Adelaar RS, Nunley JA, Myerson MS, et al. (1994) Clinical Rating Systems for the Ankle-Hindfoot, Midfoot, Hallux, and Lesser Toes. Foot Ankle Int 15: 349-353. [Crossref]

16. Budiman-Mak E, Conrad KJ, Roach KE (1991) The Foot Function Index: a measure of foot pain and disability. J Clin Epidemiol 44: 561-570. [Crossref]
17. Venditto T, Tognolo L, Rizzo RS, Iannuccelli C, Di Sante L, et al. (2015) 17-Italian Foot Function Index with numerical rating scale: Development, reliability, and validity of a modified version of the original Foot Function Index. Foot 25: 12-18. [Crossref]

18. Hosseinzadeh P, Kelly DM, Zionts LE (2017) Management of the relapsed clubfoot following treatment using the Ponseti method. J Am Acad Orthop Surg 25: 195-203. [Crossref]

19. Thomas HM, Sangiorgio SN, Ebramzadeh E, Zionts LE (2019) Relapse rates in patients with clubfoot treated using the ponseti method increase with time: A systematic review. JBJS Rev 7: 1-10. [Crossref]

20. Stouten JH, Besselaar AT, Van Der Steen MC (2018) Identification and treatment of residual and relapsed idiopathic clubfoot in 88 children. Acta Orthop 89: 448-453. [Crossref]

21. El-Sayed M (2013) Ilizarov external fixation for management of severe relapsed clubfeet in older children. Foot Ankle Surg 19: 177-181. [Crossref]

22. Gupta P, Bither N (2013) Ilizarov in relapsed clubfoot: a necessary evil? J Pediatr Orthop B 22: 589-594. [Crossref]

Copyright: (C2020 Memeo A. This is an open-access article distributed under the terms of the Creative Commons Attribution License, which permits unrestricted use, distribution, and reproduction in any medium, provided the original author and source are credited. 